УдК: 616-008.63

Матиева Л.А., Колесников А.Н.

Республиканская клиническая больница Республики Ингушетия, г. Назрань ГОО ВПО «Донецкий национальный медицинский университет им. М.Горького» ДНР, г.Донецк

\title{
HКИ SARS-COV-2, «ЦИТОКИНОВЫЙ ШТОРМ», «ПОСТКОВИДНЫЙ СИНДРОМ» ИЛИ НЕВЫУЧЕННЫЕ УРОКИ SARS И MERS (ОБЗОР ЛИТЕРАТУРЫ)
}

Тяжелый острый респираторный синдром (SARS), глобальное инфекционное заболевание, впервые возникшее в 21 веке, отличается внезапной вспышкой, быстрым распространением и поразительной смертностью [1]. Большое количество исследований было сосредоточено на эпидемиологии, этиологии, патологии, клинической диагностике и лечении атипичной пневмонии, а также на поражении дыхательной системы. иммунной система и пищеварительной система у пациентов с SARS [ 2..3; $4 ; 5 ; 6 ; 7$; $8 ; 9]$.

Прошедшие в целом незаметно для Европы эпидемии SARS и MERS (атипичные пневмонии, свиной и птичий грипп) были куда более агрессивными, с несравненно большим коэффициентом репродукции и летальности. По аналогии с SARS [10] не исключено, что SARS-CoV-2 вызывает гипофизит или поражает гипоталамус за счет отека и дегенерации нейронов, тем более что случаи энцефалита при COVID-19 уже описаны. Можно предполагать, что снижение адаптационных резервов за счет гипоталамо-гипофизарной дисфункции (возможно, вследствие энцефалита), гипофизита, создаст в клиническом отношении модель сочетания первичного гипотиреоза (транзиторного, деструктивного, в том числе с транзиторной тиреотоксической фазой аутоиммунного) с центральным гипокортицизмом. Их сочетание может объяснять длительный период реконвалесценции и формирование неспецифических эндокринных симптомов и синдромов при выздоровлении, которые имели место при так называемом post-SARS-sickness syndrome.

Высказывается гипотеза, что некоторые аминокислотные последовательности вирусов SARS-CoV, как и вируса гриппа, имеют молекулярное сходство с АКТГ, 39 Матиева Л.А. и соавт. и так называемая «иммуноинвазивная стратегия» вируса реализуется благодаря этому сходству в снижении выброса кортизола в ответ на стресс в организме подвергшегося инфекционной атаке человека. Кроме того, антитела к вирусу приобретают и способность перекрестно инактивировать адренокортикотропный гормон (АКТГ) [11]. Большинство белков SARS-CoV-2 имеют 95-100\% гомологию с белками молекулы SARS-CoV, что позволяет допустить способность SARS-CoV-2 включать те же механизмы молекулярной мимикрии [12], и больной с тяжелой формой COVID-19 может быть в группе риска так называемой «глюкокортикоидной недостаточности критически тяжелого больного», что, по всей видимости, коррелирует с измененной функцией гипоталамо-гипофизарно-надпочечниковой оси, поскольку доказано, что показатели соотношения между сывороточным кортизолом и воспалительными цитокинами (количество ИЛ-6 и фактора некроза опухоли (ФНО) может достигать уровня, в 10 раз превышающего нормальные значения) намного выше у здоровых людей, чем у пациентов с ревматоидным артритом. Предполагается также, что относительная недостаточность надпочечников у таких пациентов может быть обусловлена нарушениями функционирования печени при метаболизме стероидных гормонов.

Следовательно, пациенты с тяжелой формой COVID-19 могут быть более склонны к развитию критической кортикостероидной недостаточности, связанной с заболеванием. Однако данных о динамике кортизола у пациентов c COVID-19 пока нет. Клиницисты должны быть бдительны в отношении возможности лежащего в основе относительного дефицита кортизола у пациентов с COVID-19. Примечательно, 
что неизбирательное использование краткосрочных высоких доз глюкокортикоидов во время вспышки атипичной пневмонии было поставлено под сомнение и не было признано универсальным. Одно недавнее исследование показало, что лечение кортикостероидами не связано со временем выведения вируса, продолжительностью пребывания в больнице или продолжительностью симптомов [13].

Неврологические проявления действительно возникают у пациентов с COVID-19 и включают, среди прочего, гипосмию. Экспрессия ACE2 поддерживающими обонятельными эпителиальными клетками может объяснить большую часть этих обонятельных симптомов [ 14]. Портал проникновения вируса в центральную нервную систему (ЦНС) остается неясным и может происходить гематогенным путем или непосредственно через решетчатую пластину. Ткани гипоталамуса и гипофиза действительно экспрессируют ACE2 и теоретически могут быть вирусными мишенями. Фактически, при аутопсии в гипоталамусе были выявлены отек и дегенерация нейронов наряду с идентификацией генома SARS. Биохимические доказательства вовлечения гипоталамо-гипофиза в атипичную пневмонию впервые были представлены Leow et al. в 2005 году. у 40\% пациентов были признаки центрального гипокортизолизма, большинство из которых (62,5\%) исчезли в течение года. Примечательно, что 87,5\% пациентов с центральным гипокортизолизмом испытали усталость и / или постуральное головокружение во время первоначального набора. У небольшого процента пациентов (5\%) также был центральный гипотиреоз. Авторы предположили возможность обратимого гипофизита или прямого повреждения гипоталамуса, которое могло привести к состоянию гипоталамо-гипофизарной дисфункции [15].

Соответственно, подозрение на центральный гипокортизолизм у переживших COVID-19, особенно у тех, кто жалуется на необъяснимую усталость, утомление, недомогание, ортостатическое головокружение, анорексию и апатию должно быть на первом месте.

Данных о поражении щитовидной железы коронавирусом крайне мало. Исследование, проведенное во время вспышки атипичной пневмонии в 2003 году, показало, что уровни Т3 и Т4 в сыворотке крови у пациентов с атипичной пневмонией были ниже по сравнению с контрольной группой как в острой фазе, так и в фазе выздоровления. Это могло просто означать лежащий в основе эутиреоидный синдром [ 16 ]. Однако исследование аутопсии пяти пациентов с SARS показало заметное разрушение фолликулярных и парафолликулярных клеток щитовидной железы [17].

Таким образом, в условиях продолжающейся пандемии эндокринное вовлечение COVID-19 остается в значительной степени неизученным. Эти данные предоставляют широкие возможности для будущих исследований. Как бы преждевременно это ни звучало, эндокринологи должны знать об этих возможностях в клинической практике, особенно при работе с людьми, пережившими COVID-19.

Недавние исследования нового коронавируса показали, что, как и его предшественники, значительная часть его симптоматики может быть объяснена синдромом системного воспалительного ответа (SIRS) или феноменом, подобному SIRS - цитокиновым штормом, [18]. Это воспаление опосредуется интерлейкинами (IL-6 и IL-8), высвобождаемыми моноцитами и макрофагами для стимуляции других моноцитов, а также В- и Т-лимфоцитов, в дополнение к хемоаттрактантному белку-1 моноцитов (МСР-1), хемокину, ответственному за трансмиграцию моноцитов через гематоэнцефалический барьер (ГЭБ) [19]. Действие интерлейкинов и лимфоцитов приводит к нейровоспалению, повышая проницаемость ГЭБ, и способствуя ещё большему проникновению вируса в мозг.

Tо есть, с 2000-х годов, вирус SARS несколько видоизменился, мутировал, но, кроме отсутствия информации у врачей, ничего нового не приобрел. SIRS - синдром известный с начала 90-х годов, является отождествлением тяжелого 
септического поражения и только индивидуальная чувствительность (генетическая предрасположенность) приводит развитию гипервоспаления (ну, или «цитокинового шторма»).

Следовательно, возникает так называемы «порочный круг», когда ковид-инфекция, проникая в мозг и снижая продукцию КРГ, усиливает проницаемость ГЭБ, способствуя ещё большему проникновения вируса в мозг. То есть, можно предположить, что, разорвав этот «порочный круг», можно уменьшить степень тяжести течения ковид-инфекции.

Вопрос: где же его разрывать, этот порочный круг?

Известно, что системные заболевания связаны с синдромом низкого уровня Т3 или заболеваниями, не связанными с щитовидной железой [20]. Ожидается, что тяжелый COVID-19 вызовет такое coстояние, особенно когда инфекция связана с лихорадкой и поражением нижних дыхательных путей. Кроме того, сообщалось, что инфекция SARS-CoV-2 влияет на нервную систему с вовлечением черепных нервов для обоняния и вкуса [21]. Необходимы дальнейшие исследования для оценки риска гипоталамита, потенциально приводящего к центральному гипотиреозу у пациентов с COVID-19 после ремиссии [22,23]. Тем не менее, мы рекомендуем рутинный скрининг на дисфункцию щитовидной железы у пациентов с острыми заболеваниями, если есть подозрения в том, что заболевание щитовидной железы способствует клинической картине.

Существует предположение о том, что эндокринологическая основа может объяснить некоторые из этих внелегочных симптомов из-за их сходства с различными гормональными и метаболическими нарушениями, которые часто имеют неспецифическую симптоматику [24]. Исследования связывают аберрации в оси гипоталамус-гипофиз-надпочечники (HРA) с психическими заболеваниями [25]. Более того, были описаны корреляции дисфункции оси гипоталамо-гипофиз-щитовидная железа (ГГТ) с некоторыми психосоматическими синдромами
Основное внимание уделяется определению наличия любых хронических осложнений оси HРA у выживших после SARS, в то время как характеристика их прогностических результатов и наличие дисфункции оси НРТ составляет его вторичную цель. Учитывая, что эндокринопатические свойства коронавируса, ассоциированного с SARS (SARS-CoV), B настоящее время являются недостаточно изученной областью, любые гормональные отклонения, выявленные в ходе этого предварительного исследования, внесут новые научные данные в медицинскую базу данных с потенциалом преобразования в клинически значимые терапевтические стратегии. для эндокринопатий, связанных с атипичной пневмонией.

Наблюдения за выжившими после атипичной пневмонии во время клинического наблюдения выявили ряд хронических внелегочных симптомов, таких как летаргия, недомогание, утомляемость, утомляемость, слабость, ортостатическое головокружение, анорексия, апатия, беспокойство и депрессия. Хотя в большинстве своем расплывчатые, неспецифические и нечеткие, эти симптомы напоминали жалобы, наблюдаемые при множестве эндокринных заболеваний, включая гипотиреоз, гипокортизолизм, тиреотоксикоз, гиперкальциемию, гипопитуитаризм и синдром Кушинга. Теоретически этот коронавирус может поражать любой орган во время фазы виремии. Поскольку некоторые формы гипофизита, тиреоидита и адреналита имеют вирусную этиологию [27., 28,29], поучительно определить эндокринопатическое воздействие SARS-CoV. В целом, эти результаты подчеркивают возможную этиологическую роль SARS-CoV как эндокринопатического фактора вызывающего обратимый гипофизит или прямой гипоталамический эффект.

Подострый тиреоидит (SAT) - это самостоятельно купирующееся воспалительное заболевание щитовидной железы, характеризующееся болью в шее, общими симптомами и дисфункцией щитовидной железы [30,31]. SAT обычно предшествует 
инфекция верхних дыхательных путей. Прямые и косвенные данные подтверждают вирусное или поствирусное происхождение этого заболевания, и многие вирусы были зарегистрированы как потенциально возбудители [32]. SAT - это самоограничивающееся воспалительное заболевание, характеризующееся системными симптомами, включая лихорадку, утомляемость, миалгию и анорексию, являются обычными [33,34]. Дисфункция щитовидной железы обычно имеет трехфазное течение (тиреотоксикоз, гипотиреоз и эутиреоз), которое обычно длится 3 месяца. Заметное повышение воспалительных маркеров (СОЭ и СРБ) является обычным явлением. Этиология и патогенез САТ до конца не изучены, но распространено мнение, что заболевание вызвано вирусной инфекцией или поствирусной воспалительной реакцией у генетически предрасположенных лиц. Многие другие вирусы, включая эпидемический паротит, аденовирус, ортомиксовирус, вирус Эпштейна-Барра, гепатит E, ВИЧ, цитомегаловирус, лихорадку денге и краснуху, также связаны с SAT [35, 36,37.].

Из-за хронологической связи SARSCoV-2 может считаться ответственным за начало SAT. Однако, на сегодняшний день в базах данных по ключевым словам «thyroid and COVID-19» и «adrenal and COVID-19», найдена сообщения, которые сами по себе содержат пока еще мало информации и в основном построены на аналогиях с ранее имевшими место вирусными инфекциями и их роли в развитии патологии оси гипоталамус-гипофиз-надпочечники и гипоталамус-гипофиз-ЩЖ [38].

Таким образом, принятие НКИ, как иммуноскомпрометированной эндокринопатии, позволит не только, возможно, снизить тяжесть проявлений заболевания, но и по-другому объяснить (понять) симптомы «постковидного синдрома», как подострого тиреоидита.

\section{Литература}

1. Lan Wei, Shen Sun, Jing Zhang, Hong Zhu, Yun Xu, Qiang Ma, Michael A. McNutt, Christine Korteweg, Jiang Gu Endocrine cells of the adenohypophysis in severe acute respiratory syndrome (SARS) //Biochem. Cell Biol. Downloaded from cdnsciencepub.com by 194.28 .17 .130 on 03/07/21 Biochem. Cell Biol. 88: 723-730 (2010) doi:10.1139/010022 Published by NRC Research Press. For personal use only.

2. Peiris, J.S., Chu, C.M., Cheng, V.C., Chan, K.S., Hung, I.F., Poon, L.L., et al. Clinical progression and viral load in a community outbreak of coronavirus-associated SARS pneumonia: a prospective study. Lancet, 361(9371): 1767-1772. doi:10.1016/S01406736(03)13412-5. PMID:12781535

3. Peiris, J.S., Lai, S.T., Poon, L.L., Guan, Y., Yam, L.Y., Lim, W., et al. Coronavirus as a possible cause of severe acute respiratory syndrome. Lancet, 361(9366): 1319-1325. doi:10.1016/S0140-6736(03)13077-2. PMID:12711465

4. Booth, C.M., Matukas, L.M., Tomlinson, G.A., Rachlis, A.R., Rose, D.B., Dwosh, H.A., et al. 2003. Clinical features and short-term outcomes of 144 patients with SARS in the greater Toronto area. JAMA, 289(21): 28012809. doi:10.1001/jama. 289.21.JOC30885. PMID:12734147

5. Wong, W.W., Chen, T.L., Yang, S.P., Wang, F.D., Cheng, N.C., Kuo, B.I., et al. 2003. Clinical characteristics of fatal patients with severe acute respiratory syndrome in a medical center in Taipei. J. Chin. Med. Assoc. 66(6): 323-327. PMID:12889500

6. Nicholls, J.M., Poon, L.L., Lee, K.C., Ng, W.F., Lai, S.T., Leung, C.Y., et al. 2003. Lung pathology of fatal severe acute respiratory syndrome. Lancet, 361(9371): 1773-1778. doi:10.1016/S0140-6736(03)13413-7. PMID:12781536

7. Gu, J., Gong, E., Zhang, B., Zheng, J., Gao, Z., Zhong, Y., et al. 2005. Multiple organ infection and the pathogenesis of SARS. J. Exp. Med. 202(3): 415-424. doi:10.1084/ jem.20050828.

8. Shi, X., Gong, E., Gao, D., Zhang, B., Zheng, J., Gao, Z., et al. 2005. Severe acute respiratory syndrome associated coronavirus is detected in intestinal tissues of fatal cases. Am. J. Gastroenterol. 100(1): 169176. doi:10.1111/j.1572-0241.2005.40377.x. PMID:15654797

9. Zhan, J., Deng, R., Tang, J., Zhang, B., 
Tang, Y., Wang, J.K., et al. 2006. The spleen as a target in severe acute respiratory syndrome. FASEB J. 20(13): 2321-2328. doi:10.1096/ fj.06-6324com. PMID:17077309

10. Leow MK, Kwek DS, $\mathrm{Ng} \mathrm{AW,} \mathrm{et} \mathrm{al.}$ Hypocortisolism in survivors of severe acute respiratory syndrome (SARS). Clin Endocrinol (Oxf). 2005;63(2):197-202. https://doi. org/10.1111/j.1365-2265.2005.02325.x.

11. Wheatland R. Molecular mimicry of ACTH in SARS-implications for corticosteroid treatment and prophylaxis. Med Hypotheses. 2004;63(5):855-862. https://doi. org/10.1016/j.mehy.2004.04.009.

12. Xu J, Zhao S, Teng $T$, et al. Systematic comparison of two animal-tohuman transmitted human coronaviruses: SARS-CoV-2 and SARS-CoV. Viruses. 2020;12(2):244. https://doi.org/10.3390/ v12020244.

13. Zha L, Li S, Pan L, Tefsen B, Li Y, French $\mathrm{N}$, et al. (2020) Corticosteroid treatment of patients with coronavirus disease 2019 (COVID-19). Med J Aust [Internet] [cited 2020 Apr 22]; Available from: https ://doi.wiley .com/10.5694/mja2.50577. Accessed 22 Apr 2020

14. Brann $\mathrm{DH}$, Tsukahara $\mathrm{T}$, Weinreb $\mathrm{C}$, Logan DW, Datta SR (2020) Non-neural expression of SARS-CoV-2 entry genes in the olfactory epithelium suggests mechanisms underlying anosmia in COVID-19 patients [Internet] [cited $2020 \mathrm{Apr}$ 2]. Available from: https ://biorx iv.org/looku p/ doi/10.1101/2020.03.25.00908

15. Leow MK-S, Kwek DS-K, Ng AWK, Ong K-C, Kaw GJ-L, Lee LS-U (2005) Hypocortisolism in survivors of severe acute respiratory syndrome (SARS). Clin Endocrinol (Oxf) 63:197-202

16. De Jongh F, Jobsis A, Elte J (2001) Thyroid morphology in lethal non-thyroidal illness: a post-mortem study. Eur J Endocrinol 144:221-226

17. Wei L, Sun S, Xu C, Zhang J, Xu Y, Zhu $\mathrm{H}$ et al (2007) Pathology of the thyroid in severe acute respiratory syndrome. Hum Pathol 38:95-102

18. Chen C. Advances in the research of cytokine stormmechanism induced by Corona Virus Disease 2019 and the corresponding immunotherapies. Zhonghua Shao Shang Za
ZaZhi. 2020;36(0):E005; Bell M., Taub D., Perry $\mathrm{V}$. Overriding the brain's intrinsic resistance to leukocyte recruitment with intraparenchymal injections of recombinant chemokines. Neuroscience. 1996;74(1):283-292

19. Kaplanski G. IL-6 and IL-8 production from cultured human endothelial cells stimulated by infection with Rickettsia conorii via a cell-associated IL-1 alpha-dependent pathway. J. Clin. Invest. 1995;96(6):28392844; Safwan O. Alomari, Zaki Abou-Mrad, and Ali Bydon COVID-19 and the central nervous system. Clin Neurol Neurosurg. 2020 Nov; 198: 106116. doi: 10.1016/j. clineuro.2020.106116

20. Fliers $E$, Bianco AC, Langouche $L$ \& Boelen A. Thyroid function in critically ill patients. Lancet: Diabetes and Endocrinology 20153 816-825. (https://doi.org/10.1016/ S2213-8587(15)00225-9)

21. Lechien JR, Chiesa-Estomba CM, De Siati DR, Horoi M, Le Bon SD, Rodriguez A, Dequanter D, Blecic S, El Afia F, Distinguin L et al. Olfactory and gustatory dysfunctions as a clinical presentation of mild-to-moderate forms of the coronavirus disease (COVID-19): a multicenter European study. European Archives of Oto-Rhino- Laryngology 2020 Epub. (https://doi.org/10.1007/s00405-02005965-1)

22. Gu J, Gong E, Zhang B, Zheng J, Gao Z, Zhong Y, Zou W, Zhan J, Wang S, $X i e \mathrm{Z}$ et al. Multiple organ infection and the pathogenesis of SARS. Journal of Experimental Medicine 2005202 415-424. (https:// doi.org/10.1084/jem.20050828)

23. Wei L, Sun S, Zhang J, Zhu H, Xu Y, Ma Q, McNutt MA, Korteweg C \& Gu J. Endocrine cells of the adenohypophysis in severe acute respiratory syndrome (SARS). Biochemistry and Cell Biology 201088 723-730. (https:// doi.org/10.1139/010-022)

24. Frohman, L.A. \& Felig, P. (2001) The clinical manifestations of endocrine disease. In: P. Felig, L.A. Frohman eds.Endocrinology andMetabolism, 4th edn. McGraw-Hill, New York, 19-28

25. Kathol, R.G., Noyes, R. \& Lopez, A. (1988) Similarities in hypothalamicpituitary-adrenal axis activity between patients with panic disorderand those experiencing external tress. Psychiatric 
26. Kjellman, B.F., Thorell, L.H., Orhagen, T., d'Elia, G. \& Kagedal, B.(1993) The hypothalamic-pituitary-thyroid axis in depressive patients and healthy subjects in relation to the hypothalamic-pituitaryadrenal axis. Psychiatry Research , 47, 7-21. 27. Sellmeyer, D.E. \& Grunfeld, C. (1996) Endocrine and metabolic disturbances in human immunodeficiency virus infection and the acquired immune deficiency syndrome. Endocrine Reviews, 17, 518-532

28. Hautala, T., Sironen, T., Vapalahti, O., Paakko, E., Sarkioja, T., Salmela, P.I., Vaheri, A., Plyusnin, A. \& Kauma, H. (2002) Hypophyseal hemorrhage and panhypopituitarism during Puumala Virus Infection: Magnetic Resonance Imaging and detection of viral antigen in the hypophysis.Clinical Infectious Diseases , 35, 96-101

29. Parmar, R.C., Bavdekar, S.B., Sahu, D.R., Warke, S. \& Kamat, J.R. (2001) Thyroiditis as a presenting feature of mumps. Pediatric Infectious Disease Journal, 20, 637-638

30. Nishihara $E$, Ohye $H$, Amino $N$, et al. Clinical characteristics of 852 patients with subacute thyroiditis before treatment. Intern Med. 2008;47(8):725-729

31. Benbassat CA, Olchovsky D, Tsvetov G, Shimon I. Subacute thyroiditis: clinical characteristics and treatment outcome in fiftysix consecutive patients diagnosed between 1999 and 2005. J Endocrinol Invest. 2007;30(8):631-635

32. Desailloud R, Hober D. Viruses and thyroiditis: an update. Virol J. 2009;6:5

33. Nishihara $\mathrm{E}$, Ohye $\mathrm{H}$, Amino $\mathrm{N}$, et al. Clinical characteristics of 852 patients with subacute thyroiditis before treatment. Intern Med. 2008;47(8):725-729

34. Benbassat CA, Olchovsky D, Tsvetov G, Shimon I. Subacute thyroiditis: clinical characteristics and treatment outcome in fiftysix consecutive patients diagnosed between 1999 and 2005. J Endocrinol Invest. 2007;30(8):631-635

35. Assir MZ, Jawa A, Ahmed HI. Expanded dengue syndrome: subacute thyroiditis and intracerebral hemorrhage. BMC Infect Dis. 2012;12:240

36. Bouillet B, Petit JM, Piroth L, Duong M, Bourg JB. A case of subacute thyroiditis associated with primary HIV infection. Am J
J Med. 2009;122(4):e5-e6

37. Marthnez-Artola Y, Poncino D, Garcна ML, Munnй MS, Gonzбlez J, Garcha DS. Acute hepatitis $E$ virus infection and association with a subacute thyroiditis. Ann Hepatol. 2015;14(1):141-142

38. Трошина Е.А., Мельниченко Г.А., Сенюшкина Е.С., Мокрышева Н.Г. Адаптация гипоталамо-гипофизарно-тиреоидной и гипоталамо-гипофизарно-надпочечниковой систем к новому инфекционному заболеванию - COVID-19 в условиях развития COVID-19-пневмонии и/или цитокинового шторма// Клиническая и экспериментальная тиреоидология. 2020;16(1):21-27. https://doi.org/10.14341/ket12461

Сведения об авторах

Матиева Л.А. Аспирант кафедры анестезиологии реаниматологии и неонатологии ГОО ВПО «Донецкий национальный медицинский университет им.М.Горького». Адрес: пр. Ильича, 16, г. Донецк, 83003.

Колесников А.Н. - д.мед.наук, профессор, зав.кафедрой анестезиологии, реаниматологии и неонатологии ГОО ВПО «Донецкий национальный медицинский университет им.М.Горького». Адрес: пр. Ильича, 16, г. Донецк, 83003. email: contact@dnmu.ru 